\title{
Environmental contamination with Toxocara eggs: a quantitative approach to estimate the relative contributions of dogs, cats and foxes, and to assess the efficacy of advised interventions in dogs
}

\author{
Rolf Nijsse ${ }^{1 *+}$, Lapo Mughini-Gras ${ }^{1,2+}$, Jaap A. Wagenaar ${ }^{1,3}$, Frits Franssen ${ }^{2}$ and Harm W. Ploeger ${ }^{1}$
}

\begin{abstract}
Background: Environmental contamination with Toxocara eggs is considered the main source of human toxocariasis. The contribution of different groups of hosts to this contamination is largely unknown. Current deworming advices focus mainly on dogs. However, controversy exists about blind deworming regimens for $>6$-month-old dogs, as most of them do not actually shed Toxocara eggs. We aim to estimate the contribution of different non-juvenile hosts to the environmental Toxocara egg contamination and to assess the effects of different Toxocara-reducing interventions for dogs.

Methods: A stochastic model was developed to quantify the relative contribution to the environmental contamination with Toxocara eggs of household dogs, household cats, stray cats, and foxes, all older than 6 months in areas with varying urbanization degrees. The model was built upon an existing model developed by Morgan et al. (2013). We used both original and published data on host density, prevalence and intensity of infection, coprophagic behaviour, faeces disposal by owners, and cats' outdoor access. Scenario analyses were performed to assess the expected reduction in dogs' egg output according to different deworming regimens and faeces clean-up compliances. Estimates referred to the Netherlands, a country free of stray dogs.

Results: Household dogs accounted for $39 \%$ of the overall egg output of $>6$-month-old hosts in the Netherlands, followed by stray cats (27\%), household cats (19\%), and foxes (15\%). In urban areas, egg output was dominated by stray cats (81\%). Intervention scenarios revealed that only with a high compliance (90\%) to the four times a year deworming advice, dogs' contribution would drop from 39 to $28 \%$. Alternatively, when $50 \%$ of owners would always remove their dogs' faeces, dogs' contribution would drop to $20 \%$.

Conclusion: Among final hosts of Toxocara older than 6 months, dogs are the main contributors to the environmental egg contamination, though cats in total (i.e. both owned and stray) transcend this contribution. A higher than expected compliance to deworming advice is necessary to reduce dogs' egg output meaningfully. Actions focusing solely on household dogs and cats are unlikely to sufficiently reduce environmental contamination with eggs, as stray cats and foxes are also important contributors.
\end{abstract}

Keywords: Toxocara, Eggs, Dogs, Cats, Foxes, Contribution, Contamination, Environment, Deworming, Clean-up

\footnotetext{
* Correspondence: e.r.nijsse@uu.nl

${ }^{\dagger}$ Equal contributors

'Department of Infectious Diseases and Immunology, Faculty of Veterinary

Medicine, Utrecht University, P.O. box 80.165, 3508 TD Utrecht, The

Netherlands

Full list of author information is available at the end of the article
} 


\section{Background}

Ocular and visceral larva migrans, as well as exacerbation of asthmatic allergies, are often associated with Toxocara spp. infection in humans [1-3]. This is supported by evidence from serological studies [2], although conclusive diagnosis can be very difficult [4] and seroconversion occurs often in people without recognized clinical symptoms [5].

Environmental contamination with Toxocara eggs is believed to be the main source of human infections, which are usually caused by accidental ingestion of infective eggs present in the environment. Of the different Toxocara species, Toxocara canis and Toxocara cati are considered to pose the highest zoonotic risk. Although there are incidental reports of Toxocara vitulorum [6], this species is not thought to be of significant epidemiological importance for human toxocariasis in the Netherlands. Therefore, in order to reduce the environmental contamination with Toxocara eggs, one should focus on the main egg shedders of T. canis and T. cati, i.e. dogs, cats, or foxes. Of these, dogs are probably the population of hosts in which Toxocara infections can be controlled the best by the owners, because, in contrast to cats, there is no notable population of stray dogs in the Netherlands.

The actual contribution of household dogs to the environmental contamination with Toxocara eggs is largely unknown, and so are the contributions of foxes and (either owned or un-owned) cats, which are commonly present in the Netherlands. A model quantifying the relative contributions of different final hosts to the environmental contamination with Toxocara eggs in the city of Bristol, UK [7], revealed that dogs, especially those in the age group of $<12$ weeks, were responsible for most of the total Toxocara egg output, even if it was assumed that $75 \%$ of the produced eggs did not reach the environment directly due to confinement of dogs at such a young age. Morgan et al. [7] further showed by simulation that the proportion of $T$. canis eggs reaching the environment is, not surprisingly, strongly dependent on the rates of removal of dog faeces by owners, but actual data about the compliance of dog owners to cleanup their dogs' faeces was not available and therefore could not be incorporated in the model. What also could not be considered in that model was the level of outdoor access of household cats, and the frequency of preferred use of the litterbox, or that foxes may have more or less access to some areas depending on their degree of urbanization. Accounting for the degree of access to different (outdoor) areas and removal of faeces is therefore likely to provide novel insights in the relative contributions of different hosts older than 6 months (hereafter referred to as non-juvenile hosts) to the environmental contamination by Toxocara eggs.
Currently, the European Scientific Counsel Companion Animal Parasites (ESCCAP) recommends to deworm adult dogs ( $>6$ months of age) at least four times a year [8] to reduce the impact of patent infections on the environmental contamination with Toxocara eggs. However, this recommendation is not well supported by evidence and, as it is voluntary, it leaves ample room for dog owners to deworm their dogs (or not) in whatever frequency they like. As it cannot be expected that owners make these decisions based on adequate knowledge of the public health issues related to patent Toxocara infections [9], modelling the expected outcome of differing deworming frequencies might help determine the extent to which efforts should be put into convincing dog owners to comply with recommended treatment strategies. Because final hosts younger than 6 months of age are unlikely to have acquired age resistance against patent infections with Toxocara spp., they are believed to contribute by far the most to the overall Toxocara egg production [7, 10-12]. Accordingly, the current deworming advice for these young animals, which is based on the prepatent periods of intra-uterine and lactogenic infection, as well as infection by ingesting embryonated eggs, should be propagated and enforced. This means that puppies are to be dewormed every 2 weeks up to the age of 8 weeks, followed by monthly deworming up to the age of 6 months. The same applies to the advice of daily clean-up and disposal of their faeces by the owners. This advice is to be communicated to owners of puppies and kittens without reservation. There is, however, controversy about the necessity of the advocated deworming regimen for dogs older than 6 months, as the majority of household dogs (>90\%) does not actually shed Toxocara eggs [9, 13-15]. Additionally, for dogs older than 6 months, a mean prepatent period to serve as a guideline for deworming individual dogs cannot be as easily defined as in puppies. Puppies will not yet have developed an age resistance. Age resistance leads to mostly somatic instead of tracheal migration of larvae hatched from infective eggs. Therefore, when dogs have built up an age resistance, infection with embryonated eggs will not usually lead to a patent infection. Instead of migrating through the lungs, larvae cumulate in the somatic tissues which results in a prolonged and unpredictable prepatent period. For this reason, the present study focussed on animals older than 6 months, for which the propagated deworming advice is arguable.

Building upon the work of Morgan et al. [7], the main aim of this study was to develop a quantitative modelling approach to estimate stochastically the relative contributions of different non-juvenile host species to the environmental contamination with Toxocara eggs. Not only the host density, prevalence and intensity of infection, 
but also the degree of access to different (outdoor) areas and removal of faeces were taken into account. A comprehensive data set was then compiled using both published and original data to quantify the relative contributions to the overall Toxocara egg output in the Netherlands of non-juvenile household dogs, foxes, owned and un-owned cats (hereafter referred to as stray cats), all older than 6 months. Another aim of this study was to assess the effects of implementing different deworming regimens and compliance to faeces clean-up policies for household dogs on the total environmental contamination with Toxocara eggs.

\section{Methods}

\section{Modelling approach}

Our modelling approach builds upon an existing model [7] to quantify the number of Toxocara eggs released into the environment by non-juvenile ( $\geq 6$ month-old) final hosts (dogs, household cats, stray cats, and foxes) in the Netherlands. As there are virtually no stray dogs in the Netherlands [15], only the contribution of household dogs to the environmental contamination with Toxocara eggs was quantified. Conversely, both stray and household cats were considered.

The computational method used to estimate the overall daily egg output of non-juvenile dogs, household cats, stray cats and foxes (hereafter referred to interchangeably as hosts) in the Netherlands was the same for each of these hosts, with some adaptations depending on the data available and biological characteristics of the host in question (see section Description of the model). Since degree of urbanization and age are major determinants of host population size and frequency of egg shedding hosts $[7,9,12,16,17]$, the degree of urbanization and the age structure were expected to have a strong effect on the estimates. Therefore, for all hosts, the daily egg output was estimated separately for young adults (6-12 months of age) and adults ( $>12$ months of age), and for urban $\left(>2500\right.$ addresses $/ \mathrm{km}^{2}$ ), intermediate (500-2500 addresses $\left./ \mathrm{km}^{2}\right)$ and rural $\left(<500\right.$ addresses $\left./ \mathrm{km}^{2}\right)$ areas. The age categorization was based on a previous study [9] reporting a significantly higher risk of shedding Toxocara eggs in 6-12 month-old dogs compared to older age groups. The degree of urbanization, expressed in addresses $/ \mathrm{km}^{2}$ at the postal code area level, was based on the official categorization of the Dutch Central Bureau of Statistics used in other studies in the Netherlands, e.g. $[18,19]$.

\section{Description of the model}

Let $i$ denote the host, with $i=1$ (dogs), 2 (household cats), 3 (stray cats), and 4 (foxes); let $j$ denote the age group which individuals of host $i$ belong to, with $j=1$ (young adults) and 2 (adults); and let $z$ denote the urbanization degree of the postal code area where individuals of host $i$ and age group $j$ live in, with $z=1$ (urban areas), 2 (intermediate areas), and 3 (rural areas). The expected number of Toxocara eggs per $\mathrm{km}^{2}$ released each day into the environment by host $i$ of age group $j$ living in area $z$, denoted as $E_{i j z}$, is estimated as:

$$
\begin{aligned}
& E_{i j z} \sim \operatorname{Poisson}\left(\lambda_{i j z}\right) \\
& \lambda_{i j z}=D_{i j z} \times P_{i j z} \times F_{i} \times I_{i j}
\end{aligned}
$$

where $D_{i j z}$ is the overall density (individuals $/ \mathrm{km}^{2}$ ) of host $i$ and age group $j$ living in area $z ; P_{i j z}$ is the true prevalence of patent Toxocara infections among individuals of host $i$ and age group $j$ living in area $z ; F_{i}$ is the average daily faecal output (grams of faeces per individual per day) of host $i$ released into the environment; and $I_{i j}$ is the average intensity of infection, expressed as eggs per gram of faeces (EPG), in host $i$ and age group $j$. Full details on the estimation and data sources of these parameters are reported in Table 1. A sum of the egg outputs over age groups and areas, weighted by the size of the areas themselves $\left(a_{z}\right.$, expressed in $\left.\mathrm{km}^{2}\right)$, gives the overall daily egg output of host $i$ in the Netherlands, denoted by:

$$
E_{i}=\sum_{j} \sum_{z} E_{i j z} \times a_{z}
$$

The model was based on a Monte Carlo simulation implemented in @Risk (Palisade Corp., USA) by setting 10,000 iterations with the Latin hypercube sampling technique and a seed of one. Model convergence was monitored to check how statistics changed on the output distributions. Convergence testing was enabled every 100 iterations. Default convergence options were used, with a convergence tolerance of $3 \%$ and a confidence interval of $95 \%$; all models showed optimal convergence.

\section{Data sources and model parameterization Dogs}

The density of dogs by age group and urbanization degree $\left(D_{1 j z}\right)$ was obtained from a study on the pet population in the Netherlands in 2011 included in a report compiled by the University of Applied Sciences of Den Bosch and the Council of Animal Affairs in the Hague, the Netherlands, under the mandate of the Dutch Ministry of Economic Affairs, Agriculture and Innovation [20]. Toxocara egg prevalence in dog faeces by age group and urbanization degree $\left(p_{1 j z}\right)$ was obtained from a large study on the prevalence, risk factors and owners' attitude towards deworming for Toxocara based on 916 dogs of $\geq 6$ months of age that was conducted in the Netherlands between July 2011 and August 2012 [9]. Dog owners voluntarily participated in this study and agreed on publication of the anonymised data. Such prevalence 
Table 1 Model parameters and sources, as used in the model. Parameter means are shown in Table 3

\begin{tabular}{|c|c|c|c|}
\hline Parameter & Description & Estimation & Source \\
\hline \multicolumn{4}{|l|}{ Dogs } \\
\hline$D_{1 j z}$ & Density of dogs of age group $j$ in area $z$ & Data & {$[20]$} \\
\hline$P_{1 j z}$ & $\begin{array}{l}\text { Prevalence of Toxocara patent infection in dogs of age group } \\
j \text { in area } z\end{array}$ & $=p_{1 j z} \times c_{1 j z}$ & See below \\
\hline$p_{1 j z}$ & $\begin{array}{l}\text { Coprological prevalence of Toxocara egg shedding dogs of } \\
\text { age group } j \text { in area } z\end{array}$ & $\begin{array}{l}\sim \text { Beta }\left(a_{1 j z}+1, b_{1 j z}+1\right), \text { where: } a_{1,1,1}=2, b_{1,1,1}=47 ; a_{1,2,1}=5, b_{1,2,1}=129 ; a_{1,1,2}=10, b_{1,1,2} \\
=122 ; a_{1,2,2}=12, b_{1,2,2}=389 ; a_{1,1,3}=6, b_{1,1,3}=43 ; a_{1,2,3}=7, b_{1,2,3}=137\end{array}$ & [9] \\
\hline$c_{1 j z}$ & $\begin{array}{l}\text { Proportion of dogs of age group } j \text { in area } z \text { that do not } \\
\text { display a coprophagic behaviour }\end{array}$ & $\begin{array}{l}\sim \text { Beta }\left(a_{1 j z}+1, b_{1 j z}+1\right), \text { where: } a_{1,1,1}=26, b_{1,1,1}=22 ; a_{1,2,1}=80, b_{1,2,1}=54 ; a_{1,1,2}=56, b_{1,1,2} \\
=75 ; a_{1,2,2}=226, b_{1,2,2}=175 ; a_{1,1,3}=29, b_{1,1,3}=18 ; a_{1,2,3}=89, b_{1,2,3}=55\end{array}$ & [9] \\
\hline$F_{1 j z}$ & $\begin{array}{l}\text { Average faecal output of a dog of age group } j \text { released daily } \\
\text { into the environment of area } z\end{array}$ & $=f_{1} \times s_{1 j z}$ & See below \\
\hline$f_{1}$ & Average faecal output of a dog & $\sim$ Pert $(21,254,1074)$ & {$[22-33]$} \\
\hline$s_{1 j z}$ & $\begin{array}{l}\text { Proportion of dog owners that do not comply to dog waste } \\
\text { clean-up policies for dogs of age group } j \text { in area } z\end{array}$ & $\begin{array}{l}\sim \text { Beta }\left(a_{1 j z}+1, b_{1 j z}+1\right), \text { where: } a_{1,1,1}=20, b_{1,1,1}=28 ; a_{1,2,1}=80, b_{1,2,1}=54 ; a_{1,1,2}=87, b_{1,1,2} \\
=44 ; a_{1,2,2}=277, b_{1,2,2}=154 ; a_{1,1,3}=27, b_{1,1,3}=20 ; a_{1,2,3}=106, b_{1,2,3}=37\end{array}$ & [9] \\
\hline$I_{1 j}$ & Infection intensity (EPG) for dogs of age group $j$ & $I_{1,1} \sim$ Poisson $(341.2) ; I_{1,2} \sim$ Poisson (163.7) & [34] \\
\hline \multicolumn{4}{|l|}{$\begin{array}{l}\text { Household } \\
\text { cats }\end{array}$} \\
\hline$D_{2 j z}$ & Density of household cats of age group $j$ in area $z$ & Data & {$[20]$} \\
\hline$P_{2 j z}$ & $\begin{array}{l}\text { Prevalence of Toxocara patent infection in household cats of } \\
\text { age group } j \text { in area } z\end{array}$ & $\begin{array}{l}\sim \text { Beta }\left(a_{2 j z}+1, b_{2 j z}+1\right), \text { where: } a_{2,1,1}=0, b_{2,1,1}=2 ; a_{2,2,1}=0, b_{2,2,1}=18 ; a_{2,1,2}=2, b_{2,1,2}=15 \\
a_{2,2,2}=8, b_{2,2,2}=52 ; a_{2,1,3}=2, b_{2,1,3}=1 ; a_{2,2,3}=5, b_{2,2,3}=12\end{array}$ & [Nijsse, unpublished data] \\
\hline$F_{2 j z}$ & $\begin{array}{l}\text { Average faecal output of a household cat of age group } j \\
\text { released daily into the environment of area } z\end{array}$ & $=f_{2} \times O_{2 j z}$ & See below \\
\hline$f_{2}$ & Average faecal output of a household cat & $\sim$ Pert $(10.2,19.4,52.4)$ & [35-39] \\
\hline $\mathrm{O}_{2 j z}$ & $\begin{array}{l}\text { Proportion of household cats of group } j \text { in area } z \text { with } \\
\text { outdoor access }\end{array}$ & $\begin{array}{l}\sim \text { Beta }\left(a_{2 j z}+1, b_{2 j 2}+1\right), \text { where: } a_{2,1,1}=1, b_{2,1,1}=1 ; a_{2,2,1}=5, b_{2,2,1}=13 ; a_{2,1,2}=3, b_{2,1,2}=13 ; \\
a_{2,2,2}=45, b_{2,2,2}=13 ; a_{2,1,3}=2, b_{2,1,3}=1 ; a_{2,2,3}=14, b_{2,2,3}=3\end{array}$ & [Nijsse, unpublished data] \\
\hline$I_{2 j}$ & Infection intensity (EPG) for household cats of age group $j$ & $I_{2,1} \sim$ Poisson $(372.8) ; I_{2,2} \sim$ Poisson (81.7) & {$[40]$} \\
\hline \multicolumn{4}{|l|}{ Stray cats } \\
\hline$D_{3 j z}$ & Density of stray cats of age group $j$ in area $z$ & $\sim$ Pert $(135,000,667,500,1,200,000) \times D_{2 j z} /\left(\sum_{j} \sum_{i} D_{2 j z}\right)$ & $\begin{array}{l}\text { Personal communication: } \\
\text { preliminary estimate of feral } \\
\text { cat project WUR Wageningen }\end{array}$ \\
\hline$P_{3 j}$ & $\begin{array}{l}\text { Prevalence of Toxocara patent infection in stray cats of age } \\
\text { group } j\end{array}$ & $\begin{array}{l}\sim \text { Beta }\left(a_{3 j 2}+1, b_{3 j 2}+1\right), \text { where: } a_{3,1,1}=16, b_{3,1,1}=12 ; a_{3,2,1}=17, b_{3,2,1}=8 ; a_{3,1,2}=16, b_{3,1,2} \\
=12 ; a_{3,2,2}=17, b_{3,2,2}=8 ; a_{3,1,3}=16, b_{3,1,3}=12 ; a_{3,2,3}=17, b_{3,2,3}=8\end{array}$ & [11] \\
\hline$F_{3}$ & Average faecal output of a stray cat & $\sim$ Pert $(10.2,19.4,52.4)$ & {$[35-39]$} \\
\hline$I_{3 j}$ & Infection intensity (EPG) for stray cats of age group $j$ & $I_{3,1} \sim$ Poisson (372.8); $I_{3,2} \sim$ Poisson (81.7) & [40] \\
\hline \multicolumn{4}{|l|}{ Foxes } \\
\hline$D_{4 j z}$ & Density of foxes of age group $j$ in area $z$ & $\sim$ Pert $(0.5,2.25,4) \times d_{4 j z} /\left(\Sigma_{j} \Sigma_{i} d_{4 j z}\right)$ & [41] \\
\hline$d_{4 j z}$ & Total number of foxes of age group $j$ shot in area $z$ & Data & [41] \\
\hline
\end{tabular}


Table 1 Model parameters and sources, as used in the model. Parameter means are shown in Table 3 (Continued)

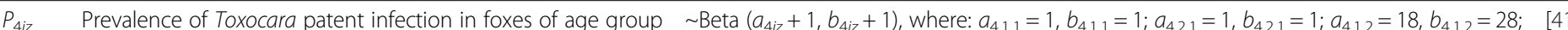

$j$ in area $z \quad a_{4,22}=9, b_{4,2,2}=12 ; a_{4,13}=57, b_{4,1,3}=74 ; a_{4,2,3}=19, b_{4,2,3}=39$

$F_{4} \quad$ Average faecal output of a fox $\quad \log \left(F_{4}\right) \sim$ Normal $(95,18)$

$I_{4 j} \quad$ Infection intensity (EPG) for foxes of age group

$I_{4,1} \sim$ Poisson (157); $I_{4,2} \sim$ Poisson (366)

Description, estimation and data sources of the model parameters used to quantify the number of Toxocara eggs released into the environment by non-juvenile ( $>6$ month-old) dogs, household cats, stray cats and foxes in the Netherlands. Parameter means are shown in Table 3 
was adjusted for the likelihood for these dogs to display coprophagic behaviour, as this causes overestimation of the true prevalence due to the passive passage of helminth eggs through the dog's digestive tract following ingestion of "egg-contaminated" faeces [21]. Coprophagy-adjusted Toxocara egg prevalence in dog faeces was estimated as $P_{1 j z}=p_{1 j z} \times c_{1 j z}$, where $p_{1 j z}$ is the observed coprological prevalence of Toxocara eggs in dogs of age group $j$ living in area $z$, and $c_{1 j z}$ is the corresponding age-and areaspecific proportion of dogs that do not display a coprophagic behaviour as provided by Nijsse et al. [9]. Both $p_{1 j z}$ and $c_{1 j z}$ parameters were modelled as Beta distributions (see Table 1).

The average faecal output of a (Dutch) dog, denoted as $f_{1}$, was derived by calculating the pooled, sample sizeweighted mean faecal output (expressed as grams of faeces per kilogram of dog's live body weight), over 12 different studies on dog food digestibility [22-33], weighted by the average bodyweight of a Dutch dog being $21.5 \mathrm{~kg}$ [20]. Minimum and maximum faecal outputs were derived proportionally by taking the Chihuahua and the Great Dane as reference breeds for the extremes of the dog faecal output range so that $f_{1}$ could be modelled as a Pert distribution (Table 1). Dog faecal output was adjusted for age-and area-specific likelihood for dog faeces to be cleaned-up by their owners as to estimate the amount of dog faeces that is actually released into the environment $\left(F_{1}\right)$. This was estimated as $F_{1(j z)}=f_{1} \times s_{1 j z}$, where $f_{1}$ is the above mentioned average faecal output of a (Dutch) dog and $s_{1 j z}$ is the proportion of dog owners that does not comply to dog waste clean-up policies among those owning dogs of age group $j$ living in area $z$. Parameter $s_{1 j z}$ was modelled as Beta distribution (Table 1) for which priors were obtained from Nijsse et al. [9].

Infection intensity (EPG) of Toxocara in dogs by age group $\left(I_{1 j}\right)$ was obtained from Sowemimo [34] and modelled as a Poisson distribution (Table 1). This parameter did not change over degrees of urbanization, but only over age groups, as it was assumed to be a parasiterelated property in a given host, irrespective of the area that host lives in.

\section{Household cats}

The density of household cats by age group and urbanization degree $\left(D_{2 j z}\right)$ was obtained from the same source as dogs [20]. Toxocara prevalence in household cats by age group and urbanization degree $\left(P_{2 j z}\right)$ was obtained from a coprological study comprising126 owned cats in the Netherlands conducted at the Faculty of Veterinary Medicine of Utrecht University between October 2011 and February 2012 (Nijsse, unpublished data). Prevalence was modelled as Beta distribution (Table 1). All cat owners voluntarily participated in this study and agreed on publication of the anonymised data.
Similar to dogs, the average faecal output of a cat, denoted as $f_{2}$, was derived by calculating the pooled, sample size-weighted mean faecal output (grams of faeces per kilogram of cat's live body weight), over five different studies on cat food digestibility [35-39]. Minimum and maximum faecal outputs were derived proportionally by taking the Singapura and the Maine Coon as reference breeds for the extremes of the cat faecal output range so that $f_{2}$ could be modelled as a Pert distribution (Table 1). Faecal output of household cats was adjusted for the age-and area-specific likelihood for household cat faeces to be actually released into the environment because these cats have access to outdoor areas. This was estimated as $F_{2(j z)}=f_{2} \times o_{2 j z}$, where $f_{2}$ is the above mentioned average faecal output of a cat and $o_{2 j z}$ is the proportion of household cats of age group $j$ in area $z$ having outdoor access. Parameter $o_{2 j z}$ was modelled as Beta distribution (Table 1) for which priors were obtained from the results of the above mentioned study (Nijsse, unpublished data).

Similar to dogs, EPG in household cats by age group $\left(I_{2 j}\right)$ was obtained from Sowemimo [40] and modelled as a Poisson distribution (Table 1), with no changes over degrees of urbanization.

\section{Stray cats}

There were no precise data on the density of stray cats by age group and urbanization degree in the Netherlands $\left(D_{3 j z}\right)$. At the time of writing, a survey to determine the number of stray cats in the Netherlands was ongoing at Wageningen University (http://www.wageningenur.nl/nl/ project/Nederlandse-zwerfkatten-in-beeld.htm). They provided us with the most likely estimate of the stray cat population in the Netherlands based on their preliminary data. This estimate is between 135,000 and 1,200,000 stray cats. Using these priors, a Pert distribution was used to estimate the total stray cat population in the Netherlands, which was distributed over age groups and urbanization degrees based on the observed age structure and urbanto-rural gradient of household cats (Table 1). Inherent to this approach is the assumption that the stray cat population follows that of household cats in terms of both age composition and spatial distribution.

Toxocara prevalence in stray cats by age group $\left(P_{3 j}\right)$ was obtained from O'Lorcain [11] and modelled as Beta distribution (Table 1). Because of the lack of data, this parameter could not vary over degrees of urbanization, but only over age groups. The average faecal output of a stray cat was the same as that of household cats (Description of the model), but it was not adjusted for outdoor access since by definition all stray cats live outside and all their faeces is released into the environment. EPG in stray cats by age group $\left(I_{3 j}\right)$ was the same as that of household cats (Table 1). 


\section{Foxes}

There were no precise data on the density of foxes by age group and urbanization degree in the Netherlands $\left(D_{4 j z}\right)$. Franssen et al. [41] estimated an overall density of 0.5 to 4.0 foxes per $\mathrm{km}^{2}$ in the Netherlands. Using these priors, a Pert distribution was used to estimate the average fox density in the Netherlands. This was then distributed over age groups and urbanization degrees based on the age structure and urban-to-rural gradient observed in a sample of 288 shot foxes submitted by hunters for routine inspection to the Dutch National Institute for Public Health and Environment between October 2010 and April 2012 [41] (Table 1). Toxocara prevalence in foxes by age group and urbanization degree $\left(P_{4 j z}\right)$ was also obtained from Franssen et al. [41], who examined the intestine of a subset of 262 foxes for the recovery of adult worms. Prevalence was modelled as a Beta distribution (Table 1). The mean and standard deviation of the faecal output of foxes were provided by Nissen et al. [42] so that the fox faecal output $\left(F_{4}\right)$ could be modelled as a log normal distribution (Table 1). EPG in foxes by age group $\left(I_{4 j}\right)$ was obtained from Saeed et al. [12] and modelled as a Poisson distribution (Table 1), with no changes over degrees of urbanization.

\section{Scenario analysis}

Since dogs are the traditional target of control activities for Toxocara infection, different scenarios were simulated to quantify the impact of varying deworming regimens for dogs on the daily egg output of dogs in the Netherlands. These scenarios were run in parallel with those assessing the sole effect of removal of dog faeces. 16 scenarios were simulated in which four putatively advised deworming regimens (i.e. twice a year, four times a year, six times a year, and 12 times a year) were applied. For this simulation the use of short-acting deworming compounds is assumed at four different rates of compliance (i.e. 30, 50, 70 and $90 \%$ ), with an average prepatent period of 30 days [43, 44] and full efficacy of the deworming treatment. Since our model was based on real-world data, of which a subset was already used by
Nijsse et al. [9], these scenarios were simulated on top of a background of observed deworming regimens and respective compliance rates present in the Dutch dog population (i.e. twice a year: $21.0 \%$ of dogs; four times a year: $17.5 \%$ of dogs; six and 12 times a year: unknown). Another four scenarios were simulated in which the observed compliance rates to dog waste clean-up policies (see Table 2) were increased by 20, 50, 70 and $90 \%$.

\section{Results}

An estimated 84,100 (95 \% CI: 55,200-120,500) Toxocara eggs per $\mathrm{km}^{2}$ per day are shed, on average, by non-juvenile hosts ( $>6$ months) in the Netherlands. This corresponded to an average egg output of $1.46 \times 10^{6}\left(0.63 \times 10^{6}-2.76 \times\right.$ $10^{6}$ ) eggs per $\mathrm{km}^{2}$ per day in urban areas, 109,500 $(54,500-196,600)$ eggs per $\mathrm{km}^{2}$ per day in intermediate areas, and 38,200 $(21,200-61,700)$ eggs per $\mathrm{km}^{2}$ per day in rural areas.

\section{Estimated host contributions to environmental egg contamination}

Of the four putative non-juvenile hosts groups considered (dogs, household cats, stray cats, and foxes), dogs were estimated to be the most important contributor to the environmental contamination with Toxocara eggs (Fig. 1). They accounted for $39.1 \%$ of the overall daily egg output of non-juvenile hosts in the Netherlands, followed by stray cats $(27.0 \%)$, household cats (19.0\%), and foxes (14.9\%). This was in spite of the relatively low prevalence of patent Toxocara infections in dogs, but by virtue of their high population density and faecal output (Table 3), as well as low compliance of dog owners to dog waste clean-up policies (Table 2). However, when summing the contributions of household and stray cats together (46.0\%), it appeared that non-juvenile cats as a whole are the primary contributor among the considered host groups. The relatively large population size and high prevalence of egg-shedding cats, either owned or stray (Table 3), along with a high proportion of household cats with outdoor access (Table 2), meant that non-juvenile cats were estimated to be the most important source of Toxocara eggs in the

Table 2 Estimated percentages of coprophagic behaviour, clean-up behavior of owners and outdoor access of household cats

\begin{tabular}{lllll}
\hline Area & Age group & Coprophagic dogs $\left(c_{1}\right), \%$ & $\begin{array}{l}\text { percentage of dog owners that } \\
\text { never/rarely clean up feces }\left(s_{1}\right), \%\end{array}$ & $\begin{array}{l}\text { Household cats with outdoor } \\
\text { access }\left(O_{2}\right), \%\end{array}$ \\
\hline Urban & Young adults & $54.00(40.23-67.46)$ & $42.00(28.81-55.78)$ & $50.00(9.41-90.56)$ \\
Urban & Adults & $59.56(51.22-67.62)$ & $59.56(51.22-67.63)$ & $30.00(12.57-51.20)$ \\
Intermediate & Young adults & $42.86(34.59-51-32)$ & $66.17(57.93-73.93)$ & $22.22(6.80-43.41)$ \\
Intermediate & Adults & $56.33(51.46-61.13)$ & $64.20(59.63-68.65)$ & $76.67(65.26-86.38)$ \\
Rural & Young adults & $61.22(47.34-74.23)$ & $57.14(43.21-70.51)$ & $60.00(19.39-93.24)$ \\
Rural & Adults & $61.64(53.64-69.34)$ & $73.79(66.36-80.60)$ & $78.95(58.56-93.59)$ \\
\hline
\end{tabular}

Estimated mean and $95 \%$ confidence interval of the posterior distribution of the rates of dogs displaying coprophagic behaviour, percentage of dog owners that never/rarely clean up feces, and outdoor access of household cats for young adults (6-12 month-old) and adults (>12 month-old) in urban, intermediate and rural areas in the Netherlands 


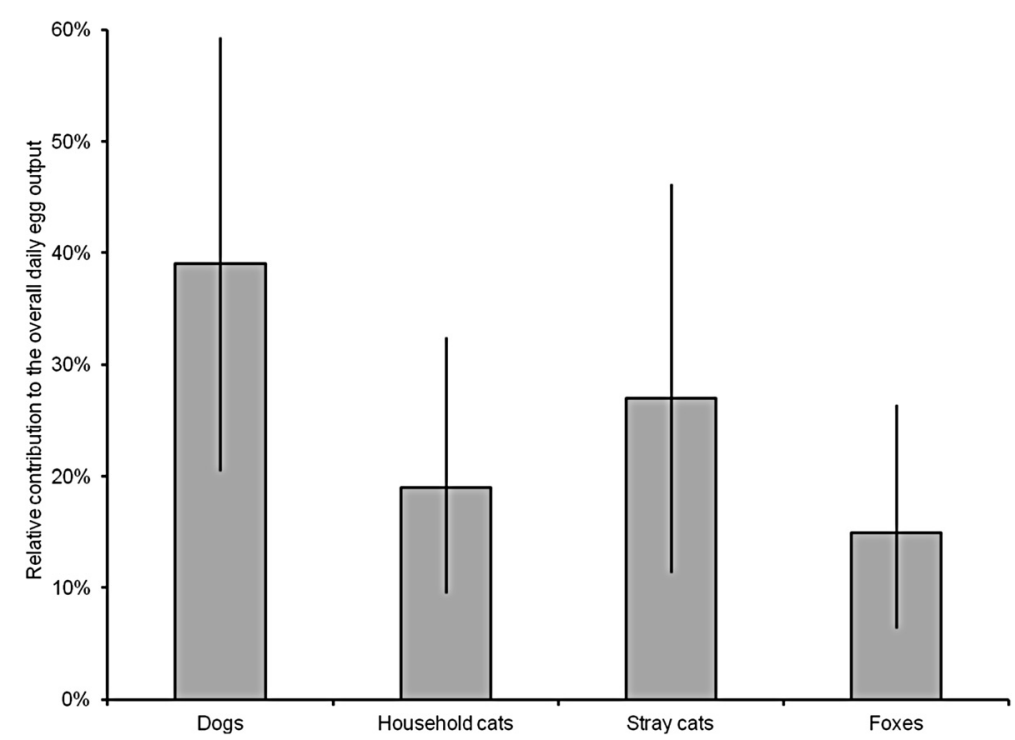

Fig. 1 Relative contributions to environmental contamination with Toxocara eggs in the whole of the Netherlands. Estimated relative contributions (\%) of non-juvenile ( $\geq 6$ month-old) dogs, household cats, stray cats, and foxes to the environmental contamination with Toxocara eggs in the whole of the Netherlands. Error bars represent $95 \%$ confidence intervals

Netherlands, despite their relatively low faecal output and intensity of infection (Table 3).

Host contributions to environmental egg contamination varied depending on the urbanization degree of the area in question (Fig. 2). In urban areas, the overall daily egg output $\left(0.97 \times 10^{9}\right.$ eggs per day, corresponding to an average of $1.46 \times 10^{6}$ eggs per $\mathrm{km}^{2}$ per day) was dominated by stray cats $(80.7 \%)$, followed by dogs (15.0\%), household cats (4.4\%), and foxes $(<0.01 \%)$. In intermediate areas, dogs were the main contributors $(54.8 \%)$ to the overall daily egg output $\left(1.48 \times 10^{9}\right.$ eggs per day, corresponding to an average of 109,500 eggs per $\mathrm{km}^{2}$ per day). In rural areas, the primary contributors to the overall daily egg output $\left(1.05 \times 10^{9}\right.$ eggs per day, corresponding to an average of 38,200 eggs per $\mathrm{km}^{2}$ per day) were foxes $(41.3 \%)$. These differences in contributions were the result of the relatively large population size of stray cats in urban areas and of foxes in rural areas, combined with a high density of dogs and household cats in intermediate areas (Table 3). Additionally, the presence of an urban-to-rural trend towards lower compliance of dog owners to dog waste clean-up policies and higher rates of outdoor access for household cats (Table 2) contributed to these differences. By contrast, foxes in urban areas and stray cats in rural areas were estimated to be few in number (Table 3), thus they appeared to contribute very little to the egg contamination in those areas.

The daily egg output of each host was dominated by adults ( $>12$ months of age) rather than young adults (612 months of age). This was in spite of the generally higher prevalence and intensity of patent Toxocara infections in younger animals, but driven by the much higher population size of the adult host populations (Table 3). Estimated contributions of adults relative to young adults of each host were $84.2 \%$ (95 \% CI: 63.3$95.7 \%$ ) for dogs, $84.7 \%$ (67.1-95.5 \%) for household cats, $84.9 \%(72.2-93.3 \%)$ for stray cats, and $69.9 \%$ (56.6-80.9\%) for foxes.

\section{Effect of deworming regimen in dogs}

The resulting estimated relative contribution to the environmental contamination of non-juvenile dogs in these different scenarios is shown in Table 4. By applying a deworming frequency of twice a year (i.e. once every 6 months), scenario analysis revealed that, compared to the current deworming frequencies applied by dog owners, the estimated percent reduction in the overall daily egg output by non-juvenile dogs in the Netherlands would vary from $3.3 \%$ (with a compliance rate of $30 \%$ ), which amounts to a $37.8 \%$ overall contribution, to $13.8 \%$ (with a compliance rate of $90 \%$ ), which amounts to an overall contribution of $33.7 \%$. With a deworming frequency of four times a year (i.e. once every 3 months), the reduction was estimated to range from $8.5 \%$ (30\% compliance) to $29.1 \%$ (90\% compliance), while a deworming regimen of six times a year (i.e. once every 2 months) would lead to an estimated reduction ranging from 13.8 (30\% compliance) to $44.1 \%$ (90\% compliance). The estimated reduction of a 12 times a year deworming regimen (i.e. once every month) would vary from 28.8 (30 \% compliance) to $89.6 \%$ (90\% compliance). 
Table 3 Estimated mean (with $95 \%$ confidence intervals) of the posterior distributions of model parameters

\begin{tabular}{|c|c|c|c|c|c|c|}
\hline & \multicolumn{2}{|l|}{ Urban areas } & \multicolumn{2}{|l|}{ Intermediate areas } & \multicolumn{2}{|l|}{ Rural areas } \\
\hline & Young adults & Adults & Young adults & Adults & Young adults & Adults \\
\hline \multicolumn{7}{|c|}{$\begin{array}{l}\text { Population density }(D), \\
\text { heads } / \mathrm{km}^{2}\end{array}$} \\
\hline $\operatorname{Dogs}^{a}$ & 9 & 208.6 & 3.4 & 79.7 & 0.4 & 8.7 \\
\hline Household cats ${ }^{a}$ & 32.5 & 755.5 & 5.7 & 131.8 & 0.5 & 12.5 \\
\hline Stray cats & $34.8(15.1-54.4)$ & $808.0(352.7-1263.8)$ & $0.3(0.1-0.5)$ & $6.9(3.0-10.9)$ & $0.01(0.006-0.02)$ & $0.3(0.1-0.5)$ \\
\hline Foxes & $0.004(0.002-0.006)$ & $0.005(0.002-0.007)$ & $0.3(0.1-0.4)$ & $0.3(0.2-0.5)$ & $0.7(0.3-1.1)$ & $0.9(0.4-1.4)$ \\
\hline \multicolumn{7}{|l|}{ Prevalence $(P), \%$} \\
\hline $\operatorname{Dogs}^{d}$ & $3.2(0.7-7.6)$ & $2.6(1.0-5.1)$ & $3.5(1.7-5.9)$ & $1.8(1.0-2.9)$ & $8.4(3.4-15.3)$ & $3.4(1.5-6.0)$ \\
\hline Household cats & $25.0(0.8-70.8)$ & $5.0(0.1-17.6)$ & $15.8(3.6-34.7)$ & $14.52(7.0-24.2)$ & $60.0(19.4-93.2)$ & $31.6(13.3-53.5)$ \\
\hline Stray cats ${ }^{c}$ & $56.7(38.9-73.6)$ & $66.7(48.2-82.8)$ & $56.7(38.9-73.6)$ & $66.7(48.2-82.8)$ & $56.7(38.9-73.5)$ & $66.7(48.2-82.8)$ \\
\hline Foxes $^{\text {b }}$ & $50.0(9.4-90.6)$ & $50.0(9.4-90.6)$ & $39.6(26.4-53.6)$ & $43.5(24.4-63.6)$ & $43.6(35.3-52.1)$ & $33.3(22.1-45.6)$ \\
\hline \multicolumn{7}{|c|}{ Faecal output (F), g/day } \\
\hline $\operatorname{Dogs}^{e}$ & $147.7(27.8-332.6)$ & $209.6(40.5-452.3)$ & $232.9(44.6-504.8)$ & $225.9(43.4-487.0)$ & $201.1(38.2-447.7)$ & $259.6(49.9-559.3)$ \\
\hline Household cats ${ }^{f}$ & $11.7(1.9-27.0)$ & $7.0(2.3-14.9)$ & $5.2(1.3-12.2)$ & $17.9(9.0-30.8)$ & $14.0(3.7-29.4)$ & $18.5(9.0-32.4)$ \\
\hline Stray cats ${ }^{9}$ & $23.4(12.1-39.5)$ & $23.4(12.1-39.5)$ & $23.4(12.1-39.5)$ & $23.4(12.1-39.5)$ & $23.4(12.1-39.5)$ & $23.4(12.1-39.5)$ \\
\hline Foxes $^{9}$ & $95.0(64.6-134.9)$ & $95.0(64.6-134.9)$ & $95.0(64.6-134.9)$ & $95.0(64.6-134.8)$ & $95.0(64.6-134.9)$ & $95.0(64.6-134.9)$ \\
\hline \multicolumn{7}{|c|}{$\begin{array}{l}\text { Infection intensity (I), } \\
\text { eggs/g faeces }\end{array}$} \\
\hline Dogsh & $341.2(305-378)$ & 163.7 (139-189) & $341.2(305-378)$ & 163.7 (139-189) & $341.2(305-378)$ & 163.7 (139-189) \\
\hline Household cats $^{\mathrm{h}}$ & $372.8(335-411)$ & $81.7(64-100)$ & $372.8(335-411)$ & 81.7 (64-100) & $372.8(335-411)$ & $81.7(64-100)$ \\
\hline Stray cats ${ }^{\mathrm{h}}$ & $372.8(335-441)$ & $81.7(64-100)$ & $372.8(335-441)$ & $81.7(64-100)$ & $372.8(335-441)$ & $81.7(64-100)$ \\
\hline Foxes $^{\text {h }}$ & $157.0(133-182)$ & $366.0(329-404)$ & $157.0(133-182)$ & $366.0(329-404)$ & $157.0(133-182)$ & $366.0(329-404)$ \\
\hline
\end{tabular}

Estimated mean and $95 \%$ confidence intervals of the posterior distribution of the host population density, prevalence of patent Toxocara infection, average daily faecal output released into the environment, and infection intensity for young adult (6-12 month-old) and adult (>12 month-old) dogs, household cats, stray cats and foxes in urban, intermediate and rural areas in the Netherlands

${ }^{a}$ Modelled deterministically as fixed single-point estimate, so no $95 \%$ confidence interval is calculated (see Table 1). ${ }^{b}$ Derived from postmortem examinations of the intestine instead of copromicroscopy. ' $\mathrm{C}$ Given the lack of detailed data, it did not change over urbanization degrees. ${ }^{\mathrm{d} A d j u s t e d ~ f o r ~ t h e ~ r a t e ~ o f ~ d i s p l a y e d ~ c o p r o p h a g i c ~}$ behaviour (see Table 2). ${ }^{\mathrm{e}}$ Adjusted for the compliance of dog owners to faeces cleaning-up policies (see Table 2). ${ }^{\mathrm{f}}$ Adjusted for the rate of outdoor access (see Table 2). ${ }^{9}$ Does not change over age groups and urbanization degrees since all stray cats and foxes release their faeces into the environment, so adjustments for outdoor access and compliance to faeces cleaning-up policies do not take place. " $D$ Does not change over urbanization degrees, but only over age groups, as it was considered as a parasite-related property of a given host, irrespective of the urbanization degree where that host live

\section{Effect of dog waste clean-up policies}

By increasing the observed compliance rates of dog owners on top of the reported waste clean-up policies (Table 2) by 20, 50, 70 and $90 \%$, the overall daily egg output of non-juvenile dogs in the Netherlands was estimated to be reduced to $32.2,20.1,12.0$ and $4.0 \%$ respectively (Table 5).

\section{Discussion}

This study presents a quantitative approach for estimating the relative contributions of different host species, all older than 6 months of age, to the environmental contamination with Toxocara eggs, accounting for host density, prevalence and intensity of infection, as well as access to different areas and removal of faeces. Moreover, we assessed the effects of enforcing different deworming regimens and compliances to faeces cleanup policies for household dogs. Both published and original data were used, using the Netherlands as an example.

Even though raw meat is considered to be an important source of human Toxocara infections in other countries [45], infection through the ingestion of embryonated eggs from the environment is by far the most important route in the Netherlands and other Western European countries $[4,15]$. Infective Toxocara eggs can survive for several years in the environment; therefore, effective measures to reduce human exposure to Toxocara should mainly aim at reducing the environmental contamination with eggs. Models like the one presented here are useful to attempt to quantify the sources of Toxocara eggs in a given locality as to prioritize control interventions and to assess the expected impact of such interventions. Morgan et al. [7] showed that the contributions of different hosts to the environmental contamination with Toxocara eggs can be quantified. Through appropriate modifications and use of 


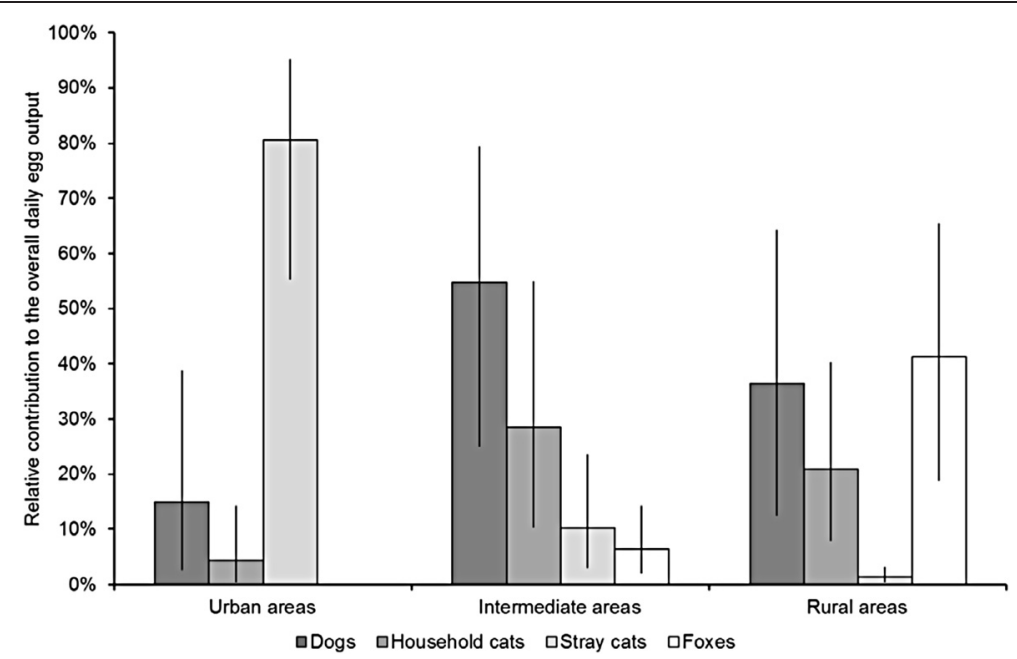

Fig. 2 Relative contributions to environmental contamination with Toxocara eggs in urban, intermediate and rural areas. Estimated relative contributions (\%) of non-juvenile ( $\geq 6$ month-old) dogs, household cats, stray cats, and foxes to the environmental contamination with Toxocara eggs in urban, intermediate and rural areas in the Netherlands. Error bars represent $95 \%$ confidence intervals

additional data, our modelling framework can be extended to other regions with different urbanization degrees and different (compositions of) definitive host populations. Actual data on reported behaviors of non-juvenile dogs, cats and their owners concerning the applied deworming regimens and (compliances to) clean-up policies are included in the model. Of course leaving out the juvenile $(<6$-month-old) group of animals, which are unlikely to have developed age resistance, meant that the largest contributors to the environmental contamination by Toxocara eggs were not considered in this analysis and that emphasis was given to the larger adult host population, for which, unlike juvenile hosts, controversy exists about the need to deworm.

Our results revealed that cats contribute the most to the environmental contamination with Toxocara eggs by nonjuvenile hosts in the Netherlands, although (household) dogs took over as the main contributors when household cats and stray cats were considered as two separate groups. This is in line with Morgan et al.'s model results
[7]. However, when areas were stratified according to their degree of urbanization, host contributions appeared to differ greatly, with stray cats dominating in urban areas, dogs dominating in intermediate areas, and foxes in rural areas. The importance of cats as a putative source of Toxocara eggs has previously been emphasized and reported to be probably underrated [4]. Our results support the notion that controlling stray cat populations should be a priority in programmes aimed at reducing the contamination of the (urban) environment with Toxocara eggs. Defining the group of hosts responsible for the majority of Toxocara eggs shed in the environment is needed to assess the extent to which the advised Toxocara-control programmes may be expected to be successful in a given locality. For instance, based on our results, it seems that increasing the deworming frequency or the rate of faeces removal for non-juvenile dogs can be expected to reach the largest proportion of shedders, and also having the largest impact especially in the intermediate areas relative to urban or rural ones.

Table 4 Estimated contribution of household dogs under different simulated deworming regimens and compliance rates

\begin{tabular}{lllll}
\hline & \multicolumn{2}{l}{ Deworming frequency (times/year) } & & \\
\cline { 2 - 5 } & $2 \times$ & $17.5 \%$ & $6 \times$ & $12 \times$ \\
\hline Baseline compliance & $21.0 \%$ & $39.1 \%$ & $39.1 \%$ & Unknown \\
Baseline contribution & $39.1 \%$ & & & $39.1 \%$ \\
Simulated compliance & & $35.8(32.9-37.6) \%$ & $33.7(29.0-36.7) \%$ & $27.8(18.0-34.4) \%$ \\
$30 \%$ & $37.8(36.6-38.5) \%$ & $33.0(27.7-36.5) \%$ & $29.7(21.5-35.0) \%$ & $19.9(3.3-30.9) \%$ \\
$50 \%$ & $36.3(33.9-37.9) \%$ & $30.4(22.8-35.5) \%$ & $25.7(14.0-33.3) \%$ & $12.0(0.0-27.5) \%$ \\
$70 \%$ & $35.0(31.4-37.3) \%$ & $27.7(17.7-34.2) \%$ & $21.9(6.7-31.7) \%$ & $4.1(0.0-24.3) \%$ \\
$90 \%$ & $33.7(29.0-36.7) \%$ & & & \\
\hline
\end{tabular}

The estimated percent contribution $(95 \% \mathrm{Cl})$ of household dogs to the overall daily Toxocara egg output under different simulated deworming regimens and compliance rates. Baseline compliance refers to the observed compliance rates according to Nijsse et al. [9] 
Table 5 Estimated contribution of household dogs under different compliance rates of cleaning-up faeces by owners

\begin{tabular}{lc}
\hline Compliance & Contribution to Toxocara egg output \\
\hline $20 \%$ & $32.2(36.4-26.7) \%$ \\
$50 \%$ & $20.1(31.2-3.1) \%$ \\
$70 \%$ & $12.0(26.1-0.0) \%$ \\
$90 \%$ & $4.0(24.3-0.0) \%$ \\
\hline
\end{tabular}

Estimated percent contribution $(95 \% \mathrm{Cl})$ of household dogs to the overall daily Toxocara egg output under different simulated compliance rates of cleaning-up dog faeces

While the degree of urbanization mirrors the extent of suitable habitat for different definitive hosts, published data on the actual habitat preferences of foxes in the Netherlands are lacking. Our assumption about the distribution of the Dutch fox population over urbanization degrees was based on the urban-to-rural gradient observed in a convenience sample of shot foxes submitted by hunters for the screening for Echinococcus multilocularis. While it is clear that fox shooting is not usually practiced in urban areas to ensure the safety of the public, it is true that foxes have only sporadically been spotted in large Dutch cities (e.g. The Hague, Amsterdam, and Rotterdam) [46]. Therefore, most foxes appear to be dispersed over rural and intermediate areas relative to urban areas, although there may be some underestimation of the actual contribution of foxes in urban areas. For stray cats, instead, we assumed that their spatial distribution would resemble that of household cats. This meant that stray cats were found to be far more abundant in urban areas. Although it is conceivable that urban areas provide plenty of shelter and food to sustain large stray cat populations, it has been reported that stray cat dispersal might differ over seasons and different types of habitats $[47,48]$. This would imply that our contribution to environmental contamination with Toxocara eggs of non-juvenile stray cats in urban areas might be overestimated due to insufficient insights in the spatio-temporal pattern of this cat population. Moreover, the population of stray cats in the Netherlands is actually composed of both feral (sylvatic) cats and, previously owned, abandoned stray cats which might prefer different habitats. Because key characteristics of landscape use of stray cats in the Netherlands are lacking and information about the actual dispersal of the stray cat population is scarce, outcomes of the model could not be differentiated further. However, in this study, the tendency of cats to dwell in areas with high availability of food and shelter has been decisive to assume the preference for urban areas. Future studies should focus on differentiating the contributions of these feline subpopulations, including their egg shedding patterns, habitat preferences, population structure, and possible contacts with humans.
Apart from the need to acquire more specific information about each host population, several other limitations in the model can be identified. As information in literature about the mean reproductive worm burden in adult hosts is lacking, our model made use of known EPG-values as a measure of the intensity of infection $[12,34,40]$. Modelling the number of egg-producing worms present in the intestines and their fecundity in animals older than 6 months would have probably been a more biologically sound approach. We speculate that this would have probably led to a reduction in the maximum number of eggs shed by large-sized dogs as the number of adult worms per host is not expected to be linearly correlated with its bodyweight, but rather with the dose of infective eggs/larvae ingested. Given the hosts we considered here, this assumption will have the largest effect on the modelled canine egg output, as the different breeds of dogs show the largest variation in bodyweight.

As mentioned earlier, we focussed on dogs older than 6 months because younger dogs are known to be Toxocara egg shedders of paramount importance [7, 10, 49]. Consensus exists that in this young age group, the propagated deworming regimen [8] and proper disposal of faeces must be enforced in any case. Conversely, the rationale of recommendations to control Toxocara infections in adult animals is much more arguable. If <6-month-old animals were included in the model, their contribution would have probably surpassed that of non-juvenile hosts, while the deworming advice for this age group would in fact remain the same.

The scenario analysis revealed that only in the case of a high compliance rate to a high deworming frequency (i.e. $\geq 50 \%$ of owners deworming their dogs 12 times a year), the contribution of non-juvenile household dogs could be expected to be halved. It is unclear what rate of voluntary compliance to a given deworming regimen would be feasible to reach in the Netherlands or in any other country. Several studies in the Netherlands have reported a compliance of circa $40 \%$ for deworming at least twice a year, but this was observed after conducting a campaign propagating deworming via the media or by asking clients visiting a veterinary clinic $[15,50]$. Customized advice for dogs frequently shedding eggs or dogs at high risk of shedding might be more efficient in reducing the contribution of non-juvenile household dogs to the environmental contamination [9]. Blind treatments at different frequencies do not appear to be as successful as may be expected $[9,13,51]$. Considering that only about $5 \%$ of non-juvenile household dogs actually are shedding Toxocara eggs at a given moment in time $[9,14,15,52]$, the question is legitimate whether it is worthwhile to invest in a policy of frequent blind treatments. The same can be said for the clean-up of 
dog faeces, though enforcement of mandatory removal of dog faces is perhaps more realistic, and our model showed that this would lead to results comparable to those that can be obtained with frequent deworming. Additional benefits (esthetical and hygienic) of the removal of dog faeces from the environment can play a decisive role in defining the priority of interventions. Both deworming and faeces removal were simulated separately, but the outcome of simulations assessing interaction effects between the different policies and compliances might differ from those assessing these effects independently of one another. It is therefore recommended that future studies assess these interactions and collect more information about incentives for dog owners to comply to one and/or to another policy. In addition, it is worth mentioning that we assumed an overall efficacy of $100 \%$ for the deworming intervention, but this might not always be the case under field circumstances. Together, these results would make the (mandatory) clean-up of faeces a more pursuable Toxocara-control option than deworming per se.

Finally, because of the different defecation behaviors of household dogs, household cats, stray cats, and foxes, and the likely differences in the longevity of Toxocara eggs in the environment associated with these behaviors, our results might not entirely reflect the origin of the eggs actually present in the environment. Our model, therefore, was only able to predict the relative contributions of different hosts to the total number of eggs released into the environment, but not to the chance of their recovery some time afterwards.

In conclusion, a quantitative model is presented with which the relative contributions of different host species to the environmental contamination with Toxocara eggs can be estimated. This model expands on the previously published model of Morgan et al. [7]. Filling in gaps in current knowledge will improve the quality of data gathered to inform the model, providing more precise evidence about the most promising targets and strategies to reduce the environmental contamination with Toxocara eggs.

\section{Consent to publish}

All the dog and cat owners voluntarily participated in this study and agreed on publication of the anonymised data.

\section{Competing interests}

The authors declare that they have no competing interests.

\footnotetext{
Authors' contributions

RN and LM were involved in writing the study design, developing the model, analysis and interpretation of the outcomes and writing and revising the manuscript. RN was involved in generating biological data concerning dogs, cats and foxes. LM was involved in constructing the model. LM and RN contributed equally in this paper. JW and HP were involved in writing the study design, critically revising the manuscript and in the interpretation of results. FF was involved in generating biological data concerning foxes, in the interpretation of these results and in critically revising the manuscript. All authors read and approved the final manuscript.
}

\section{Acknowledgements}

The authors thank Bayer Animal Health for partially financing this study. Bayer Animal Health did not have any influence on the design of this study or on the outcomes. The Veterinary Microbiological Diagnostic Centre of the Faculty of Veterinary Medicine of Utrecht University, Stephanie Rodenberg and students performing their research internship are acknowledged for their substantial contribution to the laboratory work and Nicole Buijtendijk for the administrative support. The owners participating in this study are acknowledged for supplying faecal samples of their pets and answering the questionnaire. Dr. Ibon Tamayo-Uria is acknowledged for his support in spatial data translation. The authors also like to thank Paul Overgaauw and Market Response for sharing the data of the report "Feiten en Cijfers". Francesca Neijenhuis is acknowledged for sharing the preliminary estimation of the size of the Dutch stray cat population.

\section{Author details}

'Department of Infectious Diseases and Immunology, Faculty of Veterinary Medicine, Utrecht University, P.O. box 80.165, 3508 TD Utrecht, The Netherlands. ${ }^{2}$ National Institute for Public Health and the Environment, Centre for Infectious Disease Control, P.O. box 1, Bilthoven 3720 BA, The Netherlands. ${ }^{3}$ Central Veterinary Institute of Wageningen UR, Houtribweg 39, 8221 RA Lelystad, The Netherlands.

Received: 21 April 2015 Accepted: 14 July 2015

Published online: 28 July 2015

\section{References}

1. Pinelli E, Brandes S, Dormans J, Gremmer E, van Loveren $H$. Infection with the roundworm Toxocara canis leads to exacerbation of experimental allergic airway inflammation. Clin Exp Allergy. 2008;38(4):649-58.

2. Pinelli E, Herremans T, Harms MG, Hoek D, Kortbeek LM. Toxocara and Ascaris seropositivity among patients suspected of visceral and ocular larva migrans in the Netherlands: Trends from 1998 to 2009. Eur J Clin Microbiol Infect Dis. 2011;30(7):873-9.

3. Aranzamendi C, Sofronic-Milosavljevic L, Pinelli E. Helminths: Immunoregulation and inflammatory diseases-which side are Trichinella spp. and Toxocara spp. on? J Parasitol Res. 2013;2013:329438. doi:10.1155/2013/329438.

4. Fisher M. Toxocara cati: An underestimated zoonotic agent. Trends Parasitol. 2003;19(4):167-70

5. de Melker HE, van der Peet TE, Berbers WAM, et al. Pilot-onderzoek voor het Pienter-project: Seroprevalenties voor bof, mazelen, rubella, kinkhoest, Toxoplasma gondii, Toxocara, Trichinella spiralis en hepatitis A. 213675004. Bilthoven: Dutch Institute of Public Health and the Environment; 1995. Available: http://www.rivm.nl/ dsresource?objectid=rivmp:10858\&type=org\&disposition=inline\&ns_nc=1.

6. Borgsteede FH, Holzhauer M, Herder FL, Veldhuis-Wolterbeek EG, Hegeman C. Toxocara vitulorum in suckling calves in the Netherlands. Res Vet Sci. 2012;92(2):254-6.

7. Morgan ER, Azam D, Pegler K. Quantifying sources of environmental contamination with Toxocara spp. eggs. Vet Parasitol. 2013;193(4):390-7.

8. ESCCAP, September 2010-last update, Guideline 01 2nd edition Worm Control in Dogs and Cats. Available: http://www.esccap.org/uploads/docs/ nkzqxmxn_esccapgl 1 endoguidelines.pdf.

9. Nijsse R, Ploeger HW, Wagenaar JA, Mughini-Gras L. Toxocara canis in household dogs: Prevalence, risk factors and owners' attitude towards deworming. Parasitol Res. 2014;114(2):561-9.

10. Barriga OO. A critical look at the importance, prevalence and control of toxocariasis and the possibilities of immunological control. Vet Parasitol. 1988;29(2-3):195-234.

11. O'Lorcain P. Epidemiology of Toxocara spp. in stray dogs and cats in Dublin, Ireland. J Helminthol. 1994;68(4):331-6.

12. Saeed IS, Kapel CM. Population dynamics and epidemiology of Toxocara canis in Danish red foxes. J Parasitol. 2006;92(6):1196-201.

13. Sager H, Moret C, Grimm F, Deplazes P, Doherr MG, Gottstein B. Coprological study on intestinal helminths in Swiss dogs: Temporal aspects of anthelminthic treatment. Parasitol Res. 2006;98(4):333-8.

14. Claerebout E, Casaert S, Dalemans AC, et al. Giardia and other intestinal parasites in different dog populations in northern Belgium. Vet Parasitol. 2009;161(1-2):41-6. 
15. Overgaauw PA, van Zutphen $L$, Hoek D, et al. Zoonotic parasites in fecal samples and fur from dogs and cats in the Netherlands. Vet Parasitol. 2009;163(1-2):115-22.

16. Reperant LA, Hegglin D, Fischer C, Kohler L, Weber JM, Deplazes P. Influence of urbanization on the epidemiology of intestinal helminths of the red fox (Vulpes vulpes) in Geneva, Switzerland. Parasitol Res. 2007;101(3):605-11.

17. Beugnet F, Bourdeau P, Chalvet-Monfray K, et al. Parasites of domestic owned cats in Europe: Co-infestations and risk factors. Parasit Vectors. 2014;7:291. doi:10.1186/1756-3305-7-291.

18. Mughini Gras L, Smid JH, Wagenaar JA, et al. Increased risk for Campylobacter jejuni and C. coli infection of pet origin in dog owners and evidence for genetic association between strains causing infection in humans and their pets. Epidemiol Infect. 2013;141(12):2526-35.

19. Mughini-Gras L, Enserink R, Friesema I, Heck M, van Duynhoven $Y$, van Pelt W. Risk factors for human salmonellosis originating from pigs, cattle, broiler chickens and egg laying hens: A combined case-control and source attribution analysis. PLoS One. 2014;9(2):e87933. doi:10.1371/ journal.pone.0087933.

20. HAS Den Bosch. Feiten \& cijfers - gezelschapsdierensector. 2011;ISBN 97890-817710-1-6. Den Bosch: HAS Kennistransfer, Hogeschool HAS Den Bosch. 2011. Available:http://www.hasdenbosch.nl/sites/default/files/null/Feiten\% 20\%26\%20Cijfers\%20van\%20de\%20Gezelschapsdierensector\%202011.pdf.

21. Nijsse R, Mughini-Gras L, Wagenaar JA, Ploeger HW. Coprophagy in dogs interferes in the diagnosis of parasitic infections by faecal examination. Vet Parasitol. 2014;204(3-4):304-9.

22. Cherbut $\mathrm{C}$, Ruckebusch $\mathrm{Y}$. The effect of indigestible particles on digestive transit time and colonic motility in dogs and pigs. $\mathrm{Br} J$ Nutr. 1985;53(3):549-57.

23. Diez M, Hornick JL, Baldwin P, Van Eenaeme C, Istasse L. The influence of sugar-beet fibre, guar gum and inulin on nutrient digestibility, water consumption and plasma metabolites in healthy beagle dogs. Res Vet Sci. 1998;64(2):91-6.

24. Burkhalter TM, Merchen NR, Bauer $L L$, et al. The ratio of insoluble to soluble fiber components in soybean hulls affects ileal and total-tract nutrient digestibilities and fecal characteristics of dogs. J Nutr. 2001;131(7):1978-85.

25. Grieshop CM, Flickinger EA, Fahey Jr GC. Oral administration of arabinogalactan affects immune status and fecal microbial populations in dogs. J Nutr. 2002;132(3):478-82.

26. Flickinger EA, Schreijen EM, Patil AR, et al. Nutrient digestibilities, microbial populations, and protein catabolites as affected by fructan supplementation of dog diets. J Anim Sci. 2003;81(8):2008-18.

27. Swanson KS, Kuzmuk KN, Schook LB, Fahey Jr GC. Diet affects nutrient digestibility, hematology, and serum chemistry of senior and weanling dogs. J Anim Sci. 2004;82(6):1713-24.

28. Quigley 3rd JD, Campbell JM, Polo J, Russell LE. Effects of spray-dried animal plasma on intake and apparent digestibility in dogs. J Anim Sci. 2004;82(6):1685-92.

29. Brambillasca S, Purtscher F, Britos A, Repetto JL, Cajarville C. Digestibility, fecal characteristics, and plasma glucose and urea in dogs fed a commercial dog food once or three times daily. Can Vet J. 2010;51(2):190-4.

30. Vasupen K, Yuangklang C, Beynen AC, Einerhand AWC. Effect of dietary polydextrose on feces consistency and macronutrient digestibility in healthy dogs. Am J Anim Vet Sci. 2011;6(3):105-11.

31. Beloshapka AN, Duclos LM, Vester Boler BM, Swanson KS. Effects of inulin or yeast cell-wall extract on nutrient digestibility, fecal fermentative endproduct concentrations, and blood metabolite concentrations in adult dogs fed raw meat-based diets. Am J Vet Res. 2012;73(7):1016-23.

32. Sabchuk TT, Félix AP, Comin JG, Alarça LG, de Oliveira SG, Maiorka A Digestibility and behavior of dogs housed in kennels or metabolic cages. R Bras Zootec. 2012;41(1):118-22.

33. Brambillasca S, Britos A, Deluca C, Fraga M, Cajarville C. Addition of citrus pulp and apple pomace in diets for dogs: Influence on fermentation kinetics, digestion, faecal characteristics and bacterial populations. Arch Anim Nutr. 2013;67(6):492-502.

34. Sowemimo OA. Prevalence and intensity of Toxocara canis (Werner, 1782) in dogs and its potential public health significance in Ile-lfe, Nigeria. J Helminthol. 2007:81(4):433-8.

35. Hesta M, Hoornaert E, Verlinden A, Janssens GP. The effect of oligofructose on urea metabolism and faecal odour components in cats. J Anim Physio Anim Nutr (Berl). 2005;89(3-6):208-14.
36. Barry KA, Wojcicki BJ, Middelbos IS, Vester BM, Swanson KS, Fahey Jr GC. Dietary cellulose, fructooligosaccharides, and pectin modify fecal protein catabolites and microbial populations in adult cats. J Anim Sci. 2010;88(9):2978-87.

37. Vester BM, Beloshapka AN, Middelbos IS, et al. Evaluation of nutrient digestibility and fecal characteristics of exotic felids fed horse- or beef-based diets: Use of the domestic cat as a model for exotic felids. Zoo Biol. 2010;29(4):432-48.

38. Kanakupt K, Vester Boler BM, Dunsford BR, Fahey Jr GC. Effects of shortchain fructooligosaccharides and galactooligosaccharides, individually and in combination, on nutrient digestibility, fecal fermentative metabolite concentrations, and large bowel microbial ecology of healthy adults cats. J Anim Sci. 2011;89(5):1376-84.

39. Kerr KR, Beloshapka AN, Morris CL, et al. Evaluation of four raw meat diets using domestic cats, captive exotic felids, and cecectomized roosters. J Anim Sci. 2013;91(1):225-37.

40. Sowemimo OA. Prevalence and intensity of gastrointestinal parasites of domestic cats in Ode - Irele and Oyo communities, southwest Nigeria. J Parasitol Vector Biol. 2012;4(1):7-13.

41. Franssen F, Nijsse R, Mulder J, et al. Increase in number of helminth species from Dutch red foxes over a 35-year period. Parasit Vectors. 2014;7:166. doi:10.1186/1756-3305-7-166.

42. Nissen S, Thamsborg SM, Kania PW, Leifsson PS, Dalsgaard A, Johansen MV Population dynamics and host reactions in young foxes following experimental infection with the minute intestinal fluke. Haplorchis pumilio Parasit Vectors. 2013;6:4. doi:10.1186/1756-3305-6-4.

43. Dubey JP. Patent Toxocara canis infection in ascarid-naive dogs. J Parasitol. 1978;64(6):1021-23.

44. Parsons JC. Ascarid infections of cats and dogs. Vet Clin North Am Small Anim Pract. 1987;17(6):1307-39.

45. Taira K, Saeed I, Permin A, Kapel CM. Zoonotic risk of Toxocara canis infection through consumption of pig or poultry viscera. Vet Parasitol. 2004;121(1-2):115-24.

46. Muijen T. Vossen in Rotterdam. Omgaan met vossen in Rotterdam. bSR-rapport 236. Rotterdam: Bureau stadsnatuur. 2014. Available: http://www.rotterdam.nl/Clusters/Maatschappelijke\%20ontwikkeling/ Document\%202014/Sport\%20en\%20Cultuur/bSR-rapport-236Omgaan_met_vossen_in_Rotterdam.pdf

47. Horn JA, Mateus-Pinilla N, Warner RE, Heske EJ. Home range, habitat use, and activity patterns of free-roaming domestic cats. J Wildl Manage. 2011:75(5):1177-85.

48. Spotte S. Free-ranging cats: Behavior, ecology, management. 1st ed. Hoboken: Wiley; 2014.

49. Greve JH. Age resistance to Toxocara canis in ascarid-free dogs. Am J Vet Res. 1971;32(8):1185-92.

50. Overgaauw PA, Boersema JH. Assessment of an educational campaign by practicing veterinarians in the Netherlands on human and animal Toxocara infections. Tijdschr Diergeneeskd. 1996;121(21):615-18.

51. Bajer A, Bednarska M, Rodo A. Risk factors and control of intestinal parasite infections in sled dogs in Poland. Vet Parasitol. 2011;175(3-4):343-50.

52. Becker AC, Rohen M, Epe C, Schnieder T. Prevalence of endoparasites in stray and fostered dogs and cats in northern Germany. Parasitol Res. 2012;111(2):849-57.

\section{Submit your next manuscript to BioMed Central and take full advantage of:}

- Convenient online submission

- Thorough peer review

- No space constraints or color figure charges

- Immediate publication on acceptance

- Inclusion in PubMed, CAS, Scopus and Google Scholar

- Research which is freely available for redistribution 